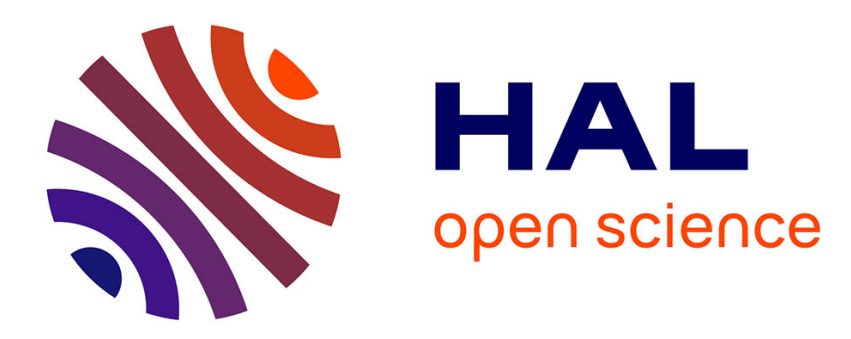

\title{
Generalized Proximal Distances for Bilevel Equilibrium Problems
}

\author{
G. Bento, J. Cruz Neto, J. Lopes, A. Soares Jr, Antoine Soubeyran
}

\section{To cite this version:}

G. Bento, J. Cruz Neto, J. Lopes, A. Soares Jr, Antoine Soubeyran. Generalized Proximal Distances for Bilevel Equilibrium Problems. SIAM Journal on Optimization, 2016, 26 (1), pp.810 - 830. 10.1137/140975589 . hal-01690192

\section{HAL Id: hal-01690192 \\ https://hal-amu.archives-ouvertes.fr/hal-01690192}

Submitted on 1 Feb 2022

HAL is a multi-disciplinary open access archive for the deposit and dissemination of scientific research documents, whether they are published or not. The documents may come from teaching and research institutions in France or abroad, or from public or private research centers.
L'archive ouverte pluridisciplinaire HAL, est destinée au dépôt et à la diffusion de documents scientifiques de niveau recherche, publiés ou non, émanant des établissements d'enseignement et de recherche français ou étrangers, des laboratoires publics ou privés. 


\title{
GENERALIZED PROXIMAL DISTANCES FOR BILEVEL EQUILIBRIUM PROBLEMS*
}

\author{
G. C. BENTO ${ }^{\dagger}$, J. X. CRUZ NETO $\ddagger$, J. O. LOPES $\ddagger$, P. A. SOARES JR. ${ }^{\ddagger}$, AND \\ A. SOUBEYRAN ${ }^{\S}$
}

\begin{abstract}
We consider a bilevel problem involving two monotone equilibrium bifunctions and we show that this problem can be solved by a proximal point method with generalized proximal distances. We propose a framework for the convergence analysis of the sequence generated by the algorithm. This class of problems is very interesting because it covers mathematical programs and optimization problems under equilibrium constraints. As an application, we consider the problem of the stability and change dynamics of a leader-follower relationship in a hierarchical organization.
\end{abstract}

Key words. equilibrium problem, bilevel problem, proximal algorithms, proximal distance, variational rationality

AMS subject classifications. 90C33, 47J25, 65K05, 91E10

DOI. $10.1137 / 140975589$

1. Introduction. Consider the problem of the stability and change dynamics of collective actions (defined as the current actions of the leader and the follower) in a hierarchical organization. This is, among others, a crucial point for the dynamics of organizations in economics and management sciences. At a higher level, there is a large body of literature about stability and change dynamics, starting in the field of economics with Schumpeter in [1,2], and in that of management sciences with Nelson and Winter in [3], within an evolutionary perspective inspired by the theory of evolution in biology. These dynamics abound; for example, Leana and Barry [4], at the organizational level, stated that "... organizations and individual employees increasingly are pursuing change in how work is organized, how it is managed and in who is carrying it out. At the same time, there are numerous individual, organizational, and societal forces promoting stability in work and employment relations." In this article, the authors examine "change and stability and the forces driving individuals and organizations to pursue both," considering that "some level of tension between stability and change is an inevitable part of organizational life...". Hoping to solve this very important problem related to the survival and dynamic efficiency of organizations, the most important step is to embed this problem in a larger one. We respond to this dynamic collective action problem in the following way: we first propose a simple model of collective actions in a hierarchical organization. Then, we use the recent variational rationality approach presented by Soubeyran in $[5,6]$ as a required enlarged framework to model and unify a large amount of worthwhile stability and change dynamics, which end in variational traps.

At the mathematical level, given a nonempty subset $K$ of $\mathbb{R}^{n}$ and $f, h: K \times K \rightarrow \mathbb{R}$, two bifunctions satisfying the property $f(x, x)=0, h(x, x)=0$, for all $x \in K$, the

\footnotetext{
* Received by the editors July 2, 2014; accepted for publication (in revised form) January 19, 2016; published electronically March 23, 2016.

http://www.siam.org/journals/siopt/26-1/97558.html

${ }^{\dagger}$ IME, Universidade Federal de Goiás, Goiânia, GO 74001-970, BR (glaydston@ufg.br).

${ }^{\ddagger} \mathrm{CCN}$, DM, Universidade Federal do Piauí, Terezina, PI 64049-550, BR (jxavier@ufpi.edu.br, jurandir@ufpi.edu.br, pedrosoares@uespi.br).

$\S$ Aix-Marseille University (Aix-Marseille School of Economics), CNRS \& EHESS, FR (antoine. soubeyran@gmail.com).
} 
present paper considers the following bilevel equilibrium problem (BEP):

$$
\text { find } \bar{x} \in S(f, K) \text { such that } h(\bar{x}, y) \geq 0, y \in S(f, K) \text {, }
$$

where $S(f, K)=\{u \in K: f(u, z) \geq 0, z \in K\}$; see Chadli, Chbani, and Riahi [7] or, more recently, Moudafi [8], Chbani and Riahi [9]. It is worth noting that a more general formulation has appeared in Muu and Oettli [10], where the authors considered the constrained region as any closed and convex set.

1.1. The nested Stackelberg BEP formulation. In this traditional formulation, $h$ and $f$ refer to the leader's (head) and follower's payoffs, which can have several different interpretations. In economics, the famous Stackelberg model of a hierarchical (nested) relation between a leader " $h$ " and a follower " $f$ " is a sequential model of choice, within a given current period; see [11]. It supposes that, in the first stage of this period, the leader chooses an individual action $y^{h} \in X^{h}$ and that, in the second stage, the follower provides a best response $y^{f} \in R^{f}\left(y^{h}\right) \subset X^{f}$. The current collective action of the leader and the follower is $y=\left(y^{h}, y^{f}\right) \in X=X^{h} \times X^{f}$. As a best response, the follower chooses an action $y^{f}$ among those maximizing his gain $g^{f}\left(y^{h}, y^{f}\right)$ (minimizing his loss $-g^{f}\left(y^{h}, y^{f}\right)$ ), given the individual action $y^{h} \in X^{h}$ chosen first by the leader, i.e., $g^{f}\left(y^{h}, y^{f}\right) \geq g^{f}\left(y^{h}, z^{f}\right)$ for all $z^{f} \in X^{f}$. If the leader knows the best response function of the follower (for example, if he knows the payoff function of the follower, $g^{f}: X \rightarrow \mathbb{R}$, which associates with each $x=\left(x^{h}, x^{f}\right)$ the number $\left.g^{f}\left(x^{h}, x^{f}\right)=g^{f}(x)\right)$, and if the follower is only able to provide a well-determined, for example, unique, best response, then, in the first stage, the leader can anticipate the best response $y^{f} \in R^{f}\left(y^{h}\right)$ of the follower, for every action $y^{h}$ he chooses to perform in the first stage. Then, the leader solves the nested bilevel program,

$$
\max \left\{g^{h}\left(y^{h}, y^{f}\right): y^{f} \in R^{f}\left(y^{h}\right), y^{h} \in X^{h}\right\},
$$

where

$$
y^{f} \in R^{f}\left(y^{h}\right) \Longleftrightarrow g^{f}\left(y^{h}, y^{f}\right) \geq g^{f}\left(y^{h}, z^{f}\right), z^{f} \in X^{f} .
$$

The drawback of this formulation is the possible multiplicity of the follower's best response.

There is a close relationship between the Stackelberg model and the point raised by Dempe and Dutta in [12], namely, "Is bilevel programming a special case of a mathematical program with complementarity constraints?"

We advocate that the previous problem refers to a special way to solve the indeterminacy problem of the leader within the Stackelberg model. More precisely, the above problem is related to the indeterminacy problem of the leader who only knows the best response corresponding to the follower $x \rightarrow R(x)$, where $R(x)$ is the subset of feasible best responses of the follower in reaction to any possible action " $x$ " performed by the leader. This is the case because the Stackelberg model supposes that the leader only knows, ex ante, before performing an action, both his optimization program [12, equation 1.2] and of the follower [12, equation 1.1], parametrized by his own action $x$. Hence, if, for some $x$, the follower optimization program has several solutions (which belong to $R(x)$, i.e., if $R(x)$ is a subset and not a point), the leader will have, in the absence of additional information, to select in an ad hoc way the best response $y \in R(x)$. There are different ad hoc ways to select $y$ within $R(x)$ :

- the behavioral approach: to choose either the optimistic or the pessimistic solution; 
- the computational solution: to replace the subset $R(x)$ of the follower's best responses by the KKT first order necessary conditions of the follower optimization program, say the $K K T$ subset $K(x)$. This is a way to enlarge $\mathrm{R}(\mathrm{x})$ to enable the selection of a solution from this larger, but much easier to characterize, set $K(x)$. Then, when, for example, convexity assumptions are carried out for the follower program, a $y$ in $K(x)$ belongs to $R(x)$ (necessary conditions are sufficient). Then, if $K(x)$ is reduced to a point, so is $R(x)$, and the leader has a selection procedure, which is much easier to compute. The trick is that the leader can impose conditions on $K(x)$ to obtain a unique best response, from each $x$.

1.2. The variational BEP formulation. Let us consider the variational rationality (VR) approach (see $[5,6]$ ), where agents, starting from an initial uncomfortable position, prefer to move and to follow an acceptable transition to finally end in a trap. Then, the variational BEP formulation (see Chadli, Chbani, and Riahi [7] and Moudafi [8]) refers to the end part $\bar{x}$ of a VR story, where an acceptable transition between the starting position and the ends is hidden.

Let us focus the attention on the last period of this transition, before reaching a trap in the current and final periods. Let $x=\left(x^{h}, x^{f}\right) \in K$ and $y=\left(y^{h}, y^{f}\right) \in K$ be the past and current collective actions of agents $h$ and $f$, where agent $h$ carries out the past and current actions $x^{h}$ and $y^{h}$. Let $g^{h}(x)$ and $g^{f}(x)$ be the past gains (to be increased) of agents $h$ and $f$. Let $g^{h}(y)$ and $g^{f}(y)$ be their current gains. Then, there is a loss for agent $f$ to change from the past collective action $u$ to the current collective action $z$ if $f(u, z)=g^{f}(u)-g^{f}(z) \geq 0$. If this is true for all $z \in K$, then $u$ represents an optimal position (as a collective action) for agent $f$. The subset $S(f, K)=\{u \in K: f(u, z) \geq 0, z \in K\}$ represents the set of stationary traps of the follower (optimum, equilibrium, stable position,... without inertia in this simple case), where, being in one of these positions $u \in S(f, K)$, the follower prefers to stay there than to move to any different position $z \in K$. Suppose that, in the first stage of the current period, as a leader (head) agent $h$ can narrow the choice of collective actions of the follower $f$ to his subset of stationary traps $S(f, K)$. Then, the leader will be certain that, whichever stationary trap that the follower will select, the follower will not try to move again. If the leader chooses, from his point of view, the best follower stationary trap from among all of them, say $\bar{x} \in S(f, K)$, then, the process will end in $\bar{x}$, because, in this situation, $f$ will prefer to stay than to move. Given that the follower will not move, neither does the leader. This is the end of this VR story.

In this model, the leader $h$ first chooses the set chosen by the follower, namely, $S(f, K)$, and a position (collective action) $\bar{x} \in S(f, K)$ within this set. Then, the leader and the follower perform the collective action. This seems to show that the variational BEP formulation is very different from the Stackelberg nested BEP formulation; however, this is not the case. Let us examine why. A comparison of Stackelberg's and Moudafi's formulations requires us to define the Stackelberg BEP formulation in terms of collective actions. Let

$$
B=\left\{y=\left(y^{h}, y^{f}\right), y^{f} \in R^{f}\left(y^{h}\right)\right\}
$$

be the subset of collective actions that defines the best response map of the leaderfollower relationship. Then, the nested Stackelberg BEP formulation is

$$
\max \left\{g^{h}(y), y \in B\right\} .
$$

This means that the leader $h$ chooses the best collective action among the subset of 
best response collective actions for the follower. The program

$$
\bar{x} \in \arg \max \left\{g^{h}(y), y \in B\right\}
$$

means that $g^{h}(\bar{x}) \geq g^{h}(y)$ for all $y \in B$. This shows that, for the leader, there is a loss $h(\bar{x}, y)=g^{h}(\bar{x})-g^{h}(y) \geq 0$ to move from the collective best response action $\bar{x} \in B$ to any other best response collective action $y \in B$. On his side, if, in the first stage, after having first proposed the collective action $\bar{x}=\left(\bar{x}^{h}, \bar{x}^{f}\right) \in S(f, K)$ the leader first carries out his individual action $\bar{x}^{h}$, then the follower will be better equipped to give his best response $\bar{x}^{f} \in R^{f}\left(\bar{x}^{h}\right)$. Then, $f(\bar{x}, y)=g^{f}(\bar{x})-g^{f}(y) \geq 0$ for all $y=\left(\bar{x}^{h}, y^{f}\right)$.

Thus, the two formulations, although different, are very similar. The BEP variational formulation seems to be more interesting for two reasons. First, it escapes from the best response problem, where, in the case of the multiplicity of best responses, $R^{f}\left(y^{h}\right) \subset X^{f}$ being a subset, the leader does not know which best response $y^{f} \in R^{f}\left(y^{h}\right)$ the follower will provide. Then, the traditional solution is to choose an ad hoc selection $y^{f} \in R^{f}\left(y^{h}\right)$. Second, the BEP variational formulation is well adapted to a VR dynamic and behavioral formulation, which allows the hidden acceptable transition to be made explicit. Although it allows starting from an initial uncomfortable position, it approaches and reaches the desired end.

In mathematics, the BEP has been widely studied and is a very active field of research; see, for instance, Ding in [13], Anh, Khanh, and Van in [14], Chen, Wan, and Cho in [15] and references therein. One of the motivations is that it covers optimization problems and mathematical programs with equilibrium constraints. These problems were addressed by Luo, Pang, and Ralph in [16] and Migdalas, Pardalos, and Varbrand in [17]. Bilevel problems were first formalized as optimization problems in the early 1970s by Bracken and McGill in [18].

Some authors have presented iterative processes to approximate a solution for bilevel problems. Cabot in [19] has built an hierarchical algorithm capable of minimizing several functions over their successive solution sets. Facchinei et al. in [20] considered centralized and distributed algorithms for the numerical solution of a hemivariational inequality where the feasible set is given by the intersection of a closed convex set with the solution set of a lower-level monotone variational inequality.

Moudafi in [8] adapted to BEP the proximal algorithm generalized by himself to equilibrium problems in [21]. In his convergence analysis the author used the following assumption

$$
\left\|x^{k+1}-x^{k}\right\|=o\left(\varepsilon_{k}\right) .
$$

Given the difficulty of verifying this assumption, the author himself has left as an open problem the "to remove condition (1.2)." As far as we know, Chbani and Riahi in [9] provide an answer to this question, by replacing assumption (1.2) by the following geometric property:

$$
(\mathcal{H}): \quad \sum_{k=1}^{\infty} \lambda_{k} \varepsilon_{k}\left[f_{z}^{*}\left(\frac{2 p}{\varepsilon_{k}}\right)-\sigma_{S(f, K)}\left(\frac{2 p}{\varepsilon_{k}}\right)\right]<\infty, z \in S(f, K), p \in \mathcal{R}\left(\mathcal{N}_{S(f, K)}\right),
$$

where $\mathcal{R}\left(\mathcal{N}_{S(f, K)}\right)$ denotes the range of $\left.\mathcal{N}_{S(f, K)}\right)$ (see section 6 for the definition of the other elements involved). This condition has previously appeared, for instance, in Attouch, Czarnecki, and Peypouquet [22, 23], where the authors have confirmed its validity in some situations. It is worth highlighting that Van Dinh and Dung Muu in [24] also studied a penalty method for BEPs, thereby also avoiding (1.2). 
In this paper, under the hypothesis of monotonicity, we present a proximal algorithm with generalized distances for BEPs. One of the reasons for using generalized distances instead of Euclidean norms is that the calculations and equations can be greatly simplified by an appropriate choice of a generalized distance that allows us to explore the geometry of the constraints. It is important to note that the hypotheses required for the good definition of our algorithm, namely, the hypotheses of Theorem 5.5, hold for a double regularization (see Silva and Eckstein [25] for more details about this regularization), because $\nabla_{1} d(\cdot, y)$ is strongly monotone with modulus $\mu>0$, which implies assumption ( $L 5)$ (see section 5.1). Hence, some assumptions that were required for Bregman distances become superfluous. A broad explanation about generalized proximal distances is provided by Auslender and Teboulle [26], Burachik and Dutta [27] and references therein. We point out that our algorithm retrieves and generalizes the proximal point method for BEPs presented in [8].

The organization of our paper is as follows. In section 2, we present an informal presentation of the VR approach. In sections 3,4 , and 5 we define equilibrium bifunctions, proximal distances, and regularized bifunctions and their behavioral interpretations as advantages or losses to change functions, inconveniences to change functions, and payoffs that are worthwhile to change. In section 6 , we consider a proximal point algorithm with generalized proximal distances to solve BEPs and we derive a convergence analysis. In the last section we present an application of the stay and change dynamic of a hierarchical organization as a routinization process which ends in a routine.

\section{An informal presentation of the VR approach.}

2.1. The VR approach: An informal presentation. To be able to make a strict parallel between the mathematical part of the paper and the application of our work in behavioral sciences, we provide an informal presentation of the main concepts of the VR approach (see [5, 6]), which gives a common background to a lot of different stay/stability and change dynamics. This greatly facilitates the connection, in a few words, between substantial multiple different strands of literature on stay and move dynamics in behavioral sciences (economics, management sciences, psychology, ...). It considers the dynamics $\left\{x^{0}, x^{1}, \ldots, x^{k}, x^{k+1}, \ldots, x^{*}\right\} \subset X$ of general human behavior, including a starting point $x^{0}$, a transition $\left\{x^{1}, \ldots, x^{k}, x^{k+1}, \ldots\right\}$, and some end $x^{*}$, where $X$ can be, depending on the context, a space of actions (doings), or states (havings and beings). Let $k$ and $k+1$ be the previous and current periods, $x=x^{k}$ and $y=x^{k+1}$ be the previous and current actions or states, and $x \curvearrowright y$ be the current move from the current action $x$ to a future action $y$. In the current period $k+1$, a change is such that $x^{k+1} \neq x^{k}$, whereas a stay is $x^{k+1}=x^{k}$. Initially, agents are not where they want to be, i.e., at the desired end $x^{*} \neq x^{0}$. This provides them with the motivation to change, in order to approach and perhaps reach the end. Because they must overcome a lot of resistance to change (obstacles, difficulties), agents cannot succeed in reaching the desired end in one step. Thus, they subdivide the difficulties they face into smaller tasks. They must accept following a transition, defined as a succession of intermediate positions. This transition must be acceptable, improving sufficiently (satisficing) in each step, without requiring much current sacrifices, to avoid being stuck in the middle. The VR approach formalizes what can be an acceptable transition in terms of worthwhile transitions. For that purpose, it defines, as its central building block, five concepts: "stay and change," "worthwhile change," "marginal worthwhile stay," "worthwhile transition," and "variational trap."

Informally, the VR structure of an agent functions as follows: 
(1) a worthwhile change is such that the current motivation to change of the agent is sufficiently higher, more than some given satisficing proportion $\xi=\xi_{k+1}>0$ of his current resistance to change, in the current period $k+1$;

(2) a worthwhile transition refers to a succession of worthwhile temporary stays and changes;

(3) a variational trap is such that it represents the end of a worthwhile stay and change dynamic, feasible and desirable (i.e., an aspiration point, worthwhile to reach from some or any point of the transition), and stable (not worthwhile to leave, an equilibrium);

(4) the motivation of the agent to change is the utility of his advantages to change;

(5) the resistance of the agent to change is the disutility of his inconveniences to change;

(6) advantages to change, when they are separable, refer to the difference between the current payoff (profit, pleasure) of moving to a new position and the current payoff of staying in the previous position. Losses to change can be defined in a similar way;

(7) disadvantages to change, when they are also separable, represent the difference between the costs of being able to change and the costs of being able to stay. The VR definition of costs of being able to change and costs to be able to stay is difficult. It includes a lot of different situations and merits lengthy discussions in each different discipline in behavioral sciences. This paper will consider an important specific case;

(8) when, starting from an initial position, a worthwhile transition converges to a variational trap, it both approaches and ends in this trap.

In this paper, the VR structure of each agent is, (i) linear (motivation to change is identified as being advantages to change, and resistance to change is identified as being inconveniences to change), (ii) experience dependent (advantages and inconveniences to change depend on the past experience $e(k)$ ), adaptive (satisficing worthwhile to change ratio $\xi_{k+1}>0$ and sharing rules/weights $\varepsilon_{k+1}>0$ are chosen, each period), and separable (advantages and inconveniences refer, respectively, to differences between payoffs to change and to stay and costs of being able to change and to stay).

2.2. The dual variational BEP formulation. In the context of the VR approach, the dual formulation of the variational BEP approach considers the two functions $\widetilde{f}, \widetilde{h}: K \times K \rightarrow \mathbb{R}$, such that $\widetilde{f}(x, y)=-f(x, y)$ and $\widetilde{h}(x, y)=-h(x, y)$, aiming to find $\bar{x} \in S(\widetilde{f}, K)$ such that $\widetilde{h}(\bar{x}, y) \leq 0$ for all $y \in S(\widetilde{f}, K)$, where $S(\widetilde{f}, K)=$ $\{u \in K: \widetilde{f}(u, y) \leq 0, y \in K\}$. As seen before, the dual BEP formulation is static. It helps to modelize the stability aspect of a leader-follower problem, where agents start directly from a position where they prefer staying rather than moving (this is an equilibrium problem). However, using the recent VR approach of human behavior $[5,6]$ and the VR core proactive and dynamic concept of advantages to change during the transition (and not the discouraging and static concept of losses by changing at the end), the dual formalization better facilitates the modelization of the dynamic aspects of a leader-follower problem, the transition where, during each period, being away from an equilibrium, agents achieve a balance between changing or staying, to end in a final situation in which they both prefer to stay. It allows us to focalize the attention, not only on the end of the process, but more importantly, and also, on the transition from the starting point to the end, to know how, starting from an initial position, agents approach the final equilibrium position. In this VR dynamical context, this dual BEP formulation modelizes a dynamic leader-follower problem 
within a hierarchical organization, where, during each period, the leader manages the choice of temporary stays and changes, and the follower adapts. For example, the choice of changing $y=\left(y^{h}, y^{f}\right) \in K$ refers to a collective action, where the action $y^{h}$ of the leader means that "the leader/entrepreneur acquires a new technology, trains the worker to enable it to be used, sets a wage, and sells the final product produced by the worker," and the action $y^{f}$ of the follower means that "the follower/worker uses this technology to produce the final good." In contrast, the choice of staying $x=\left(x^{h}, x^{f}\right) \in K$ would entail "to continue to use the old technology in the same way, setting the same wage, and selling the final product in the same way." Let $\widetilde{f}(x, y) \geq 0$ and $\widetilde{h}(x, y)$ be the advantages of the follower and the leader to change from the old position $x$ to the new position $y$. The subset $S(\widetilde{f}, K)$ defines the different equilibrium positions $u \in K$ of the follower, where the advantages $\widetilde{f}(u, y) \leq 0$ to change from $u$ to any other position $y \in K$ are nonpositive. Then, the dual BEP maintains that the leader chooses, among the subset $S(\widetilde{f}, K)$ of different equilibria of the follower, a follower equilibrium $\bar{x} \in S(\widetilde{f}, K)$, where, being there, he prefers to stay rather than to move to another follower equilibrium $y \in S(\widetilde{f}, K)$, because his advantages to change $\widetilde{h}(\bar{x}, y) \leq 0$, for all $y \in S(\widetilde{f}, K)$ are nonpositive.

2.3. From behavioral sciences notation to mathematics. Hereinafter, assuming that $f$ and $h$ are given as in the introduction to this paper, the advantages for the leader and the follower to change are given by: $\widetilde{f}(x, y)=-f(x, y) \geq 0$ and $\widetilde{h}(x, y)=-h(x, y) \geq 0$, whereas their losses to change are $f(x, y) \geq 0$ and $h(x, y) \geq 0$ when they are nonnegative. The weighted advantages to change for the organization and its weighted losses to change are, respectively, given by

$$
A_{e}(x, y):=-[\varepsilon f(x, y)+h(x, y)], \quad F_{e}(x, y):=\varepsilon f(x, y)+h(x, y) .
$$

We consider a structure representing a simple and linear motivation and resistance to change. In this case the utility of advantages to change and the disutility of inconveniences to change are identical to advantages and inconveniences to change, $U_{e}\left[A_{e}(x, y)\right]=A_{e}(x, y)$ and $D_{e}\left[I_{e}(x, y)\right]=I_{e}(x, y)$. Then, the worthwhile to change payoff of the organization is $\Delta_{e, \lambda}(x, y)=A_{e}(x, y)-\lambda I_{e}(x, y)=-L_{e, \lambda}(x, y)$, where $L_{e, \lambda}(x, y)=F_{e}(x, y)+\lambda I_{e}(x, y)$.

3. A simple model of a hierarchical organization. In this short section, we provide, using the VR approach, a simple hierarchical model of an organization where equilibrium bifunctions represent advantages and losses to change. The collective action of the hierarchical firm is $x=\left(x^{h}, x^{f}\right) \in K \subset X$, where $x^{h}$ means that the entrepreneur/leader acquires a technology (defined as a bundle of means and a way to use them), trains the worker to enable this technology to be used in the given way, and sets a wage, whereas $x^{f}$ means that the worker/follower works, i.e., uses this technology. The leader first chooses and then performs the action $x^{h}$. Then, the follower uses the technology by doing $x^{f} \in K\left(x^{h}\right)$ if only if $\left(x^{h}, x^{f}\right) \in K$. Let us define the revenue function $r: X \rightarrow \mathbb{R}_{+}$, the wage function $w: X \rightarrow \mathbb{R}_{+}$, and the profit function of the entrepreneur $\pi: X \rightarrow \mathbb{R}$, given by $\pi(x)=r(x)-w(x)-\rho\left(x^{h}\right)$, where $\rho\left(x^{h}\right) \geq 0$ is the costs of acquisition of technology and the costs of training the worker to enable him to use it. The worker payoff is $\omega(x)=w(x)-\delta\left(x^{f}\right) \geq 0$, where $\delta\left(x^{f}\right) \geq 0$ is the disutility of working $x^{f}$. When this is the case, the advantages to change from position $x$ to position $y$ are, for the leader and the follower, $\widetilde{h}(x, y)=$ $\pi(y)-\pi(x) \geq 0$ and $\tilde{f}(x, y)=\omega(y)-\omega(x) \geq 0$. This means that neither the profit nor the wage do not decrease, $\pi(y) \geq \pi(x)$ and $\omega(y) \geq \omega(x)$. When this is the case, the 
losses of changing from position $x$ to position $y$ are, for the leader and the follower, $h(x, y)=\pi(x)-\pi(y) \geq 0$ and $f(x, y)=\omega(x)-\omega(y) \geq 0$. Neither the profit nor the wage do not increase. In this case, the advantages to change functions are separable. Then, the BEP is simplified to find $\bar{x} \in K$ such that, $\widetilde{h}(\bar{x}, y)=\pi(y)-\pi(\bar{x}) \leq 0$ for all $y \in S(\widetilde{f}, K)$, i.e., such that $\widetilde{f}(y, z)=\omega(z)-\omega(y) \leq 0$ for all $z, y \in K$. This is equivalent to find $\bar{x} \in K$ such that $\pi(\bar{x}) \geq \pi(y)$ for all $y \in K$ such that $\omega(y) \geq \omega(z)$ for all $z \in K$.

\section{Proximal distances and inconveniences to change.}

4.1. Proximal distances. Next, we recall some definitions and results associated with the proximal and induced proximal distances that are useful in the remainder of the paper and were explained in [26, 27].

Definition 4.1. A function $d: \mathbb{R}^{n} \times \mathbb{R}^{n} \rightarrow \mathbb{R}_{+} \cup\{\infty\}$ is known as a proximal distance with respect to a closed, nonempty, and convex set $S \subset \mathbb{R}^{n}$ iff, for every fixed $y \in \operatorname{int} S$, the following properties hold:

(i) $d(\cdot, y)$ is a proper, lsc convex function and $C^{1}$ on int $S$;

(ii) $\operatorname{dom} d(\cdot, y) \subset S$, and $\operatorname{dom} \partial_{1} d(\cdot, y)=\operatorname{int} S$, where $\partial_{1} d(\cdot, y)$ denotes the classical subgradient map of the function $d(\cdot, y)$ with respect to the first variable.

Henceforth, we assume that $S \subset \mathbb{R}^{n}$ is a closed, nonempty, and convex set. The family of functions satisfying this definition is denoted by $\mathcal{D}(S)$.

The next step is to associate each given $d \in \mathcal{D}(S)$ with a corresponding proximal distance satisfying some desirable properties.

Definition 4.2. Given $d \in \mathcal{D}(S)$. Let $D: \mathbb{R}^{n} \times \mathbb{R}^{n} \rightarrow \mathbb{R}_{+} \cup\{\infty\}$ be a function such that $\operatorname{int} S \times \operatorname{int} S \subseteq \operatorname{dom} D$. Then, $D$ is referred to as the induced proximal distance to $d$ iff the following properties hold:

(H1) for every $x \in \operatorname{int} S, D(x, \cdot)$ is continuous on $\operatorname{int} S$;

(H2) $D(x, x)=0$ for all $x \in \operatorname{int} S$;

(H3) for all $x \in S$ and $\alpha \in \mathbb{R}$, the set $\{y \in \operatorname{int} S: D(x, y) \leq \alpha\}$ is bounded;

(H4) for every $x, y \in \operatorname{int} S$, it holds that

$$
\left\langle z-x, \nabla_{1} d(x, y)\right\rangle \leq D(z, y)-D(z, x)-\gamma D(x, y)
$$

for all $z \in S$ and some fixed $\gamma>0$;

(H5) if $\left\{y^{k}\right\} \subset \operatorname{int} S$ and $y^{k} \rightarrow y \in S$, then $D\left(y, y^{k}\right) \rightarrow 0$;

(H6) let $z \in S$ and $y \in \operatorname{int} S$, and take $w:=\alpha z+(1-\alpha) y$ with $\alpha \in(0,1)$. Then,

$$
D(z, w)+D(w, y) \leq D(z, y)
$$

(H7) if $\left\{x^{k}\right\},\left\{y^{k}\right\} \subset \operatorname{int} S$ are sequences such that $\left\{x^{k}\right\}$ converges to $x$ and $\left\{y^{k}\right\}$ converges to $y$, with $x \neq y$, then $\liminf _{k} D\left(x^{k}, y^{k}\right)>0$.

Remark 4.1. Properties (H1), (H2), (H3), (H5), and (H7) on generalized distances refer to more technical assumptions. Properties $(\mathrm{H} 4)$ and $(\mathrm{H} 6)$ are related to a weak form of the triangular inequality. The triangular inequality is a standard assumption in the VR approach; see [5, 6], for a strong justification.

For examples (Bregman distance, a double regularization, or a second order homogeneous proximal distance) and a thorough discussion relative to proximal and induced proximal distances see, for instance, [26, 27]. 
We denote by $\mathcal{F}(S)$ the set of pairs $(d, D)$ of generalized proximal and induced proximal distances that satisfy the conditions of Definition 4.2 , and we say that $(d, D)$ is a proximal pair associated with $S$.

As an illustrative example, we present one way to determine, for a particular choice of $\mathrm{K}$, what are the set $S$ and $(d, D) \in \mathcal{F}(S)$.

Example 4.1. Consider the set $K=\left\{x \in \mathbb{R}^{n}: g_{j}(x) \leq 0, j=1, \ldots, m\right\}$, where $g_{j}: \mathbb{R}^{n} \rightarrow \mathbb{R}$ is a convex function for $j=1, \ldots, m$. Given $\alpha>0$, define $\tilde{g}_{j}(x)=$ $g_{j}(x)-\alpha, j=1, \ldots, m$. In this case, take

$$
S=\left\{x \in \mathbb{R}^{n}:-\tilde{g}_{j}(x)>0, j=1, \ldots, m\right\} .
$$

Clearly, $K \subset$ int $S=S$. We note that, for this choice of $S$, Auslender and Teboulle in [26] defined the proper, lsc, and convex function

$$
\varphi(x)=\left\{\begin{array}{l}
\sum_{j=1}^{m}-\log \left(-\tilde{g}_{j}(x)\right) \text { if } x \in \text { int } S \\
\infty \text { otherwise }
\end{array}\right.
$$

from which the authors considered the following Bregman proximal distance associated with $\varphi_{\nu}(x):=\varphi(x)+\frac{\nu}{2}\|x\|^{2}, \nu>0$ :

$$
D_{\varphi_{\nu}}(x, y)=\sum_{j=1}^{m}\left(-\log \frac{\tilde{g}_{j}(x)}{\tilde{g}_{j}(y)}+\frac{\left\langle\nabla \tilde{g}_{j}(y), x-y\right\rangle}{\tilde{g}_{j}(y)}\right)+\frac{\nu}{2}\|x-y\|^{2} .
$$

In this case, for our choice of $S$ associated with the given set $K$ and defining $d(x, y)=$ $D(x, y):=D_{\varphi_{\nu}}(x, y)$, one has $(d, D) \in \mathcal{F}(S)$; see Burachik and Dutta [27].

Remark 4.2. Note that, from property $(H 4)$ it follows that $\nabla_{1} d(x, x)=0$.

Before presenting the method, we recall two important facts regarding generalized proximal distances verifying $(\mathrm{H} 6)$ and $(\mathrm{H} 7)$. The proofs of the following two propositions can be found in [27].

Proposition 4.3. Assume that $(d, D)$ verifies $(\mathrm{H} 6)$ and $(\mathrm{H} 7)$. If $\left\{x^{k}\right\} \subset S$ and $\left\{y^{k}\right\} \subset \operatorname{int} S$ are sequences such that

$$
\lim _{k \rightarrow \infty} D\left(x^{k}, y^{k}\right)=0
$$

and one of the sequences $\left(\left\{x^{k}\right\}\right.$ or $\left.\left\{y^{k}\right\}\right)$ converges, then the other also converges to the same limit.

Proposition 4.4. Assume that $(d, D)$ verifies $(\mathrm{H} 6)$ and $(\mathrm{H} 7)$. If $\left\{x^{k}\right\} \subset S$ and $\left\{y^{k}\right\} \subset \operatorname{int} S$ are sequences such that

$$
\lim _{k \rightarrow \infty} D\left(x^{k}, y^{k}\right)=0
$$

and that one of the sequences $\left(\left\{x^{k}\right\}\right.$ or $\left.\left\{y^{k}\right\}\right)$ is bounded, then, the following hold:

(a) The other sequence is also bounded.

(b) $\lim _{k \rightarrow \infty}\left(x^{k}-y^{k}\right)=0$. 
4.2. Inconveniences to change. Let $e=e^{k} \in E$ be the past experience of the agent in the current period $k+1$. In this work it refers to the previous collective action $e^{k}=x^{k}$. The VR rationality approach $[5,6]$ defines the inconveniences to change $I_{e}(x, y)=C_{e}(x, y)-C_{e}(x, x) \geq 0$ as the difference between the costs of being able to change $C_{e}(x, y) \geq 0$ and the costs of being able to stay $C_{e}(x, x) \geq 0$. In this paper, the costs of being able to change from the current collective action $x=x^{k+1}$ to the future collective action $y$, given the experience $e=x^{k}$ of the agents, are given by

$$
C_{e}(x, y)=\left\langle c_{e}(x), y-x\right\rangle+C_{e}(x, x)
$$

where $C_{e}(x, x)$ is the costs of being able to stay, i.e., the costs of being able to repeat the same collective action $x$ and $\left\langle c_{e}(x), y-x\right\rangle$ is the marginal costs of being able to change, i.e., the costs of being able to change the current collective action from $x$ to $y-x$. Note that, from (4.1), the inconvenience to change simplifies to

$$
I_{e}(x, y)=\left\langle c_{e}(x), y-x\right\rangle .
$$

Given the generalized distance $d\left(x^{k}, x\right)$ from the old collective action $x^{k}$ to the current one $x=x^{k+1}$, the marginal cost of being able to change are $c_{k}(x)=\nabla d_{2}\left(x^{k}, x\right)$. We have reversed the mathematical notation from $\nabla d_{1}\left(x, x^{k}\right)$ to $\nabla d_{2}\left(x^{k}, x\right)$ to emphasize that the move is from $x^{k}$ to $x=x^{k+1}$. Generalized distances are not symmetric, as required for costs of being able to change. These distances have regularity properties that are natural for the cost of being able to change. Furthermore, the costs of being able to stay $C_{e}(x, x) \geq 0$ usually do not equal zero.

\section{Reguralized bifunctions as worthwhile to change payoffs.}

5.1. The standard assumptions for equilibrium bifunctions. Mathematicians usually prefer to consider costs (losses, pains) to be of a decreasing nature, to which they apply minimization problems borrowed from physics, whereas, in behavioral sciences, researchers very often consider payoffs to involve an increase (profit, pleasure) and see them as maximization problems. Hereafter, we assume that $K \subset \mathbb{R}^{n}$ is a closed, nonempty, and convex set. To avoid losing the interest of the mathematically oriented reader, in the mathematical part of the paper we consider bifunctions $\psi: K \times K \rightarrow \mathbb{R}$, which refer to losses. Given $x, y \in K$, our basic assumptions associated with these bifunctions $\psi$ are the following:

$(L 1) \psi(x, x)=0$

$(L 2) \psi(\cdot, y): K \longrightarrow \mathbb{R}$ is upper semicontinuous;

(L3) $\psi(x, \cdot): K \longrightarrow \mathbb{R}$ is convex and lsc.

In addition to the previous assumptions, we require that $\psi$ be monotone, i.e.,

(L4) $\psi(x, y)+\psi(y, x) \leq 0$

(L5) for any sequence $\left\{y^{k}\right\} \subset K$ with $\lim _{k \rightarrow \infty}\left\|y^{k}\right\|=\infty$, there exist $u \in K$ and $k_{0} \in \mathbb{N}$ such that

$$
\psi\left(y^{k}, u\right) \leq 0, \quad k \geq k_{0} .
$$

Remark 5.1. As far as we know, assumption ( $L 5)$ was first considered by Iusem, Kassay, and Sosa in [28]. It is important to highlight that Muu and Oettli in [10] and Blum and Oettli in [29] used a condition which is sufficient for (L5), in order to establish existence results for the equilibrium problem.

TheOrem 5.1. Assume that $\psi$ satisfies (L1)-(L3) and $K$ is a compact set. Then, $S(\psi, K)$ is nonempty. 
Proof. The proof is presented by Fan in [30].

Theorem 5.2. Assume that $\psi$ satisfies (L1)-(L5). Then, $S(\psi, K)$ is nonempty.

Proof. The proof is presented by Iusem, Kassay, and Sosa in [28, Thm. 4.3].

5.2. Regularized bifunctions via generalized proximal distances. Let $f, h: K \times K \longrightarrow \mathbb{R}$ be such that assumptions (L1)-(L3) hold. Take a generalized proximal distance $d$ with respect to $S$ such that $K \subset \operatorname{int} S$. Fix $\bar{x} \in K, \lambda, \varepsilon>0$ and consider the bifunction $\bar{f}: K \times K \rightarrow \mathbb{R}$ defined by

$$
\bar{f}(x, y)=g(x, y)+\frac{1}{\lambda}\left\langle\nabla_{1} d(x, \bar{x}), y-x\right\rangle,
$$

where $g:=\varepsilon f+h$ and $\nabla_{1} d(x, \bar{x})$ denotes the gradient of $d(., \bar{x})$ at $x$. We denote by $S(\bar{f}, K)$ the solution set of the equilibrium problem associated with $\bar{f}$.

Assumption 5.1. For every sequence $\left\{y^{k}\right\} \subset K$ such that $\lim _{k \rightarrow \infty}\left\|y^{k}\right\|=\infty$,

$$
\liminf _{k \rightarrow \infty}\left[g\left(\bar{x}, y^{k}\right)+\frac{1}{\lambda}\left\langle\nabla_{1} d\left(y^{k}, \bar{x}\right), y^{k}-\bar{x}\right\rangle\right]>0 .
$$

It is worth pointing out that the last assumption appeared in Burachik and Kassay [31], where a similar proof for the next lemma can be found.

Lemma 5.3. Suppose that $f, h$ satisfy $(L 1)-(L 4)$. Then, $\bar{f}$ satisfies $(L 1)-(L 4)$. Moreover, if Assumption 5.1 holds, then $\bar{f}$ satisfies (L5).

Proof. It is clear that $\bar{f}$ satisfies $(L 1)$. Because the map

$$
y \mapsto\left\langle\nabla_{1} d(x, \bar{x}), y-x\right\rangle
$$

is convex and continuous, and $x \mapsto\left\langle\nabla_{1} d(x, \bar{x}), y-x\right\rangle$ is continuous at $x \in K$, it follows that $\bar{f}$ satisfies $(L 2)-(L 3)$. We now claim that $\bar{f}$ satisfies $(L 4)$. Indeed, from the monotonicity of $g$ and $\nabla_{1} d(\cdot, \bar{x})$, we obtain

$$
\bar{f}(x, y)+\bar{f}(y, x)=g(x, y)+g(y, x)-\frac{1}{\lambda}\left\langle\nabla_{1} d(y, \bar{x})-\nabla_{1} d(x, \bar{x}), y-x\right\rangle \leq 0 .
$$

Now, we show that $\bar{f}$ satisfies $(L 5)$. From $(L 4)$ and (5.1), we have

$$
\bar{f}\left(y^{k}, \bar{x}\right) \leq-\left[g\left(\bar{x}, y^{k}\right)+\frac{1}{\lambda}\left\langle\nabla_{1} d\left(y^{k}, \bar{x}\right), y^{k}-\bar{x}\right\rangle\right], \quad k=0,1, \ldots,
$$

and the desired result follows from Assumption 5.1.

Corollary 5.4. If $f, h$ satisfy $(L 1)-(L 4)$ and any of the following two conditions hold,

(i) $K$ is bounded,

(ii) $d$ is 1-coercive, i.e.,

$$
\lim _{\|x\| \rightarrow \infty} \frac{d(x, \bar{x})}{\|x\|}=\infty
$$

then, $\bar{f}$ satisfies (L5). 
Proof. If $K$ is bounded, then Assumption 5.1 is verified and, from Lemma 5.3, condition $(i)$ implies $(L 5)$. Let us suppose now that $(i i)$ holds and note that $\operatorname{dom} \partial g(\bar{x}, \cdot) \cap K \neq \emptyset$ (see [31, Corollary 3.1] for a detailed proof of this fact). Take $v \in \partial g(\bar{x}, \tilde{x})$, for some $\tilde{x} \in K$, and a sequence $\left\{y^{k}\right\} \subset K$ such that $\left\|y^{k}\right\| \rightarrow \infty$. Since $g(\bar{x}, \cdot)$ is convex (this follows from $(L 3)$ ), using that $v \in \partial g(\bar{x}, \tilde{x})$, we have

$$
g\left(\bar{x}, y^{k}\right) \geq g(\bar{x}, \tilde{x})+\left\langle v, y^{k}-\tilde{x}\right\rangle \geq g(\bar{x}, \tilde{x})-\|v\|\left\|y^{k}-\tilde{x}\right\|, \quad k=0,1, \ldots,
$$

where the last inequality follows from the Cauchy-Schwarz inequality. On the other hand, because $d(\cdot, \bar{x})$ is convex, we obtain

$$
d(\bar{x}, \bar{x}) \geq d\left(y^{k}, \tilde{x}\right)+\left\langle\nabla_{1} d(\cdot, \bar{x}), \bar{x}-y^{k}\right\rangle, \quad k=0,1, \ldots
$$

The last inequality combined with (5.2) yields

$g\left(\bar{x}, y^{k}\right)+\frac{1}{\lambda}\left\langle\nabla_{1} d\left(y^{k}, \bar{x}\right), y^{k}-\bar{x}\right\rangle \geq g(\bar{x}, \tilde{x})+\left\|y^{k}-\tilde{x}\right\|\left(-\|v\|+\frac{d\left(y^{k}, \tilde{x}\right)}{\left\|y^{k}-\tilde{x}\right\|}-\frac{d(\bar{x}, \bar{x})}{\left\|y^{k}-\tilde{x}\right\|}\right)$

for $k=0,1, \ldots$ Therefore, letting $k$ go to infinity in the last inequality and using that $d(\cdot, \bar{x})$ is 1-coercive, we obtain that Assumption 5.1 holds and the desired result follows again from Lemma 5.3.

Remark 5.2.

(i) If $\nabla_{1} d(\cdot, \bar{x})$ is strongly monotone with modulus $\mu>0$, i.e.,

$$
\left\langle\nabla_{1} d(y, \bar{x})-\nabla_{1} d(x, \bar{x}), y-x\right\rangle \geq \mu\|y-x\|^{2}, \quad x, y \in K,
$$

it follows that, in particular, $d(\cdot, \bar{x})$ is 1-coercive. Hence, the last corollary tells us that $\nabla_{1} d(\cdot, \bar{x})$ being strongly monotone is also a sufficient condition to hold (L5).

(ii) By choosing distance functions $d$ such that, for each $\bar{x}$, the operator $\nabla_{1} d(\cdot, \bar{x})$ is strictly monotone (but not necessarily strongly monotone), our analysis includes, for example, $d(x, y)=x \ln (x / y)+y-x$ (the entropic Bregman distance). Statisticians know it as the Kullback-Leibler information divergence and it corresponds to the barrier $\varphi(t)=t \ln t-t+1$; see, for instance, $[32,33,34,35]$ and references therein.

(iii) In the previous results we presented some sufficient conditions for (L5) to hold. We note that, even for the problem of a single level, namely, when $f \equiv 0$, there exist situations where this condition is required to ensure the existence of a solution of the penalized problem. Indeed, to illustrate this fact, let us consider the following situation: let $h: K \times K \rightarrow \mathbb{R}$ be given by $h(x, y)=x-y$, with $K=[1 / 2, \infty[$, and choose $d(x, y):=(x / y)-\ln (x / y)-1$ (Bregman distance induced by $\varphi(t):=t-\ln t+1$ ). It is easy to see that $\nabla_{1} d(x, \bar{x})=(1 / \bar{x})-(1 / x)$ is strictly monotone, but is not strongly monotone or, more generally, $d$ is not 1 -coercive. Moreover, $h$ satisfies $(L 1)-(L 4)$. We claim that, for $\bar{x}=2$ and $\lambda=3$, the penalized problem does not have a solution. To see this, note that for any $x \in K$, there is $y \in K$ such that

$$
\bar{f}(x, y)=(x-y)+\frac{1}{3}\left(\frac{1}{2}-\frac{1}{x}\right)(y-x)=-\frac{(y-x)}{3}\left(\frac{5}{2}+\frac{1}{x}\right)<0 .
$$

The next result establishes the existence and uniqueness of the solution to the equilibrium problem associated with $\bar{f}$. The proof is similar to that presented in [31, Corollary 3.2]. 
THEOREM 5.5. Under the assumptions of Lemma $5.3, S(\bar{f}, K)$ is a nonempty set. Moreover, if $\nabla_{1} d(\cdot, \bar{x})$ is strictly monotone, then $S(\bar{f}, K)$ has a unique element.

Proof. From the assumptions, Lemma 5.3 implies that $\bar{f}$ satisfies $(L 1)-(L 5)$ and, in this case, $S(\bar{f}, K) \neq \emptyset$, see $\left[28\right.$, Theorem 4.3]. Now, assume that $\nabla_{1} d(\cdot, \bar{x})$ is strictly monotone and take $x_{1}, x_{2} \in S(\bar{f}, K)$. As $\bar{f}$ satisfies $(L 4)$, we have $\bar{f}\left(x_{1}, x_{2}\right)=$ $\bar{f}\left(x_{2}, x_{1}\right)=0$ and, using (5.1) with $x=x_{1}, y=x_{2}$, we obtain

$$
0=\bar{f}\left(x_{1}, x_{2}\right)=g\left(x_{1}, x_{2}\right)+\frac{1}{\lambda}\left\langle\nabla_{1} d\left(x_{1}, \bar{x}\right), x_{2}-x_{1}\right\rangle
$$

and

$$
0=\bar{f}\left(x_{2}, x_{1}\right)=g\left(x_{2}, x_{1}\right)+\frac{1}{\lambda}\left\langle\nabla_{1} d\left(x_{2}, \bar{x}\right), x_{1}-x_{2}\right\rangle .
$$

Adding the last two inequalities, we obtain

$$
0=g\left(x_{1}, x_{2}\right)+g\left(x_{2}, x_{1}\right)-\frac{1}{\lambda}\left\langle\nabla_{1} d\left(x_{1}, \bar{x}\right)-\nabla_{1} d\left(x_{2}, \bar{x}\right), x_{1}-x_{2}\right\rangle,
$$

and the result follows by using the fact that $g$ satisfies $(L 4)$.

5.3. The worthwhile to change payoff of the organization. Let $e=\bar{x}=x^{k}$ be the current experience of the organization, which is identified by the previous collective action $x^{k}$. Let $x^{k+1}$ be the current collective action and $y=x^{k+2}$ be the future collective action. The organizational advantage to change of the organization is $A_{e, \varepsilon}(x, y)=\varepsilon \widetilde{f}(x, y)+\widetilde{h}(x, y)$, where $\varepsilon=\varepsilon_{k+1}>0$ is a sharing rule, which allows aggregation of advantages to change $\widetilde{h}(x, y)$ of the leader/entrepreneur and advantages to change $\widetilde{f}(x, y)$ of the follower/worker. The inconveniences to change of the organization are $l_{e}(x, y)=C_{e}(x, y)$. Then, the worthwhile to change payoff of the organization is $\Delta_{e, \varepsilon, \xi}(x, y)=A_{e, \varepsilon}(x, y)-\xi l_{e}(x, y)$. The opposite is the not worthwhile to change payoff

$$
\Lambda_{e, \varepsilon, \xi}(x, y)=-A_{e, \varepsilon}(x, y)+\xi l_{e}(x, y)=L_{e, \varepsilon}(x, y)+\xi l_{e}(x, y),
$$

where $L_{e, \varepsilon}(x, y)=-A_{e, \varepsilon}(x, y)=\varepsilon f(x, y)+h(x, y)$ is the loss to change function of the organization, i.e., the regularized bifunction

$$
L_{e, \varepsilon}(x, y)=\bar{f}(x, y)=\varepsilon f(x, y)+h(x, y)+\frac{1}{\lambda}\left\langle\nabla_{1} d(x, \bar{x}), y-x\right\rangle .
$$

In the current period $k+1$, these payoffs are, if $e=x^{k}, \xi_{k+1}=1 / \lambda_{k+1}, x=x^{k+1}$, and $y=x^{k+2}$,

$$
\begin{aligned}
\Delta_{e_{k}, \varepsilon_{k+1}, \xi_{K+1}}(x, y) & =A_{e_{k}, \varepsilon_{k+1}}(x, y)-\xi_{k+1} l_{e_{k}}(x, y) \\
& =\varepsilon_{k+1} \widetilde{f}(x, y)+\widetilde{h}(x, y)+\frac{1}{\lambda_{k+1}}\left\langle\nabla_{1} d\left(x, x^{k}\right), y-x\right\rangle
\end{aligned}
$$

and

$$
\Lambda_{e_{k}, \varepsilon_{k+1}, \xi_{K+1}}(x, y)=f_{k}(x, y)=\varepsilon_{k+1} f(x, y)+h(x, y)+\frac{1}{\lambda_{k+1}}\left\langle\nabla_{1} d\left(x, x^{k}\right), y-x\right\rangle .
$$

The mathematical formulation uses $\varepsilon_{k}$ and $\xi_{k}$ instead of $\varepsilon_{k+1}$ and $\xi_{k+1}$, which refer, in the behavioral context, to decisions in the current period $k+1$. 
6. Generalized proximal distance as regularization method for solving BEPs. Henceforth, unless otherwise specified, we assume that $\nabla_{1} d(\cdot, \bar{x})$ is strictly monotone, $K \subset \operatorname{int}(\operatorname{dom} d(\cdot, y))$ for all $y \in \operatorname{int} S$, and that all the assumptions of Lemma 5.3 (noting that Assumption 5.1 is also part of the set of assumptions) happen.

6.1. Proximal point algorithm. In this section, following some ideas presented by Attouch, Czarnecki, and Peypouquet in [22] and Chbani and Riahi in [9], we present an approach of the proximal point algorithm with generalized distances for BEPs, where the convergence result is obtained for monotone bifunctions.

We show the existence and unicity of a solution of the regularized problem (5.1) (see Theorem 5.5) by constructing the following algorithm for solving the bilevel problem (1.1).

Algorithm 1. Take two sequences of positive real numbers $\left\{\epsilon_{k}\right\}$ and $\left\{\lambda_{k}\right\}$ such that $\lim _{k \rightarrow \infty} \varepsilon_{k}=\infty, \liminf _{k \rightarrow \infty} \lambda_{k}>0$ and consider the bifunction

$$
f_{k}(x, y)=\varepsilon_{k} f(x, y)+h(x, y)+\frac{1}{\lambda_{k}}\left\langle\nabla_{1} d\left(x, x^{k}\right), y-x\right\rangle, \quad x, y \in K .
$$

Initialization. Choose an initial point $x^{0} \in K$.

ITERATIVE STEP. Given $x^{k}$, take as the next iterate $x^{k+1} \in K$ such that

$$
x^{k+1} \in S\left(f_{k}, K\right) .
$$

Stopping CRITERIOn. If $x^{k+1}=x^{k}$ and $x^{k} \in S(f, K), S T O P$.

Remark 6.1.

(a) When $f \equiv 0$ and $d$ is a Bregman proximal distance in (6.1), we retrieve the algorithm presented in [31] in a finite dimensional space.

(b) If $\left\{x^{k}\right\}$ terminates after a finite number of iterations, then it terminates at a solution of (1.1). Indeed, take $k$ such that $x^{k+1}=x^{k}$ and $x^{k} \in \mathrm{S}(f, K)$. Note $\nabla_{1} d\left(x^{k+1}, x^{k}\right)=0$. Moreover, as $f$ satisfies $(L 4)$, it is easy to see that $f\left(x^{k+1}, y\right)=0$ for any $y \in \mathrm{S}(f, K)$. Therefore, the claim follows from the definitions of $x^{k+1}$ and $f_{k}$.

(c) Notice that if $f \equiv 0$ in (6.1) it is sufficient to require, as a stopping criterion for Algorithm 1, that $x^{k+1}=x^{k}$.

(d) In terms of the VR approach, condition (6.2) is equivalent for each current period $k+1$, to the existence of the weak variational trap $x^{k+1}$. Indeed, combining the definition of $f_{k}$ in (6.1) with the definition of $S\left(f_{k}, K\right)$ and using (5.3) with $e=e_{k}, \varepsilon=\varepsilon_{k}, \lambda=\lambda_{k}$, we obtain $x^{k+1} \in S\left(f_{k}, K\right)$ if only if

$$
-f_{k}(x, y)=A_{e_{k}, \varepsilon_{k}}(x, y)-\xi_{k+1} I_{e_{k}}(x, y)=\Delta_{e_{k}, \varepsilon_{k}, \xi_{k+1}}(x, y) \leq 0,
$$

where $\xi_{k+1}=1 / \lambda_{k}$. Then, it is not worthwhile to change from the current position $x=x^{k+1}$ to any position $y \in K$ iff

$$
\Delta_{e_{k}, \varepsilon_{k}, \xi_{k+1}}(x, y) \leq 0, \quad y \in K .
$$

This last condition defines a weak variational trap $x=x^{k+1}$ in the current period $k+1$.

Next, we introduce a technical result on the nonnegative sequences of real numbers that is required in the subsequence analysis. 
LEMma 6.1. Let $\left(\xi_{k}\right)$ and $\left(\gamma_{k}\right)$ be nonnegative sequences of real numbers satisfying

(a) $\xi_{k+1} \leq \xi_{k}+\gamma_{k}$,

(b) $\sum_{k=0}^{\infty} \gamma_{k}<\infty$.

Then, the sequence $\left\{\xi_{k}\right\}$ converges.

Proof. The proof is presented by Polyak in [36, Lemma 9, p. 49].

In the sequel, given a closed, nonempty, and convex set $\Omega \subset \mathbb{R}^{n}$, we denote by $\delta_{\Omega}$, $\mathcal{N}_{\Omega}$, and $\sigma_{\Omega}$, respectively, the indicator function, the normal cone, and the support function associated with $\Omega$. Recall that

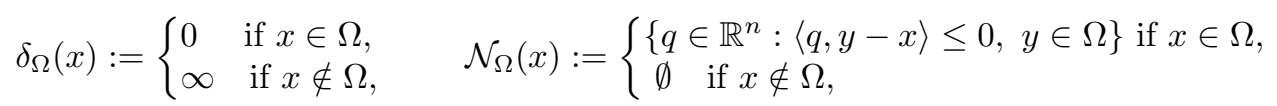

and $\sigma_{\Omega}(x)=\sup _{y \in \Omega}\langle x, y\rangle, \delta_{\Omega}^{*}=\sigma_{\Omega}$ (elements which are presented by Rockafellar in [37, Theorem 13.2, p. 114]), $\partial \delta_{\Omega}(x)=\mathcal{N}_{\Omega}(x)$, and $y \in \mathcal{N}_{\Omega}(x)$ if and only if $\sigma_{\Omega}(y)=\langle y, x\rangle$, where $\delta_{\Omega}^{*}$ denotes the conjugate function of $\delta_{\Omega}$.

Let us define the functions $f_{z}(y)=f(z, y)$ and $h_{z}(y)=h(z, y)$, for all $y \in K$.

Lemma 6.2. Take $z \in S(f, K), w \in \partial\left(h_{z}+\delta_{S(f, K)}\right)(z)$, and $p \in \mathcal{N}_{S(f, K)}(z)$ such that $w-p \in \partial h_{z}(z)$. Then,

$$
\begin{aligned}
D\left(z, x^{k+1}\right)+\frac{\lambda_{k} \varepsilon_{k}}{2} f\left(z, x^{k+1}\right) \leq & D\left(z, x^{k}\right)-\gamma D\left(x^{k+1}, x^{k}\right)+\lambda_{k}\left\langle w, z-x^{k+1}\right\rangle \\
& +\frac{\lambda_{k} \varepsilon_{k}}{2}\left[f_{z}^{*}\left(\frac{2 p}{\varepsilon_{k}}\right)-\sigma_{S(f, K)}\left(\frac{2 p}{\varepsilon_{k}}\right)\right] .
\end{aligned}
$$

Proof. From (6.1), (H4), and the monotonicity of $f$ and $h$, we have

$$
\begin{aligned}
D\left(z, x^{k+1}\right)+\frac{1}{2} \lambda_{k} \varepsilon_{k} f\left(z, x^{k+1}\right) & \\
& \leq D\left(z, x^{k}\right)-\frac{1}{2} \lambda_{k} \varepsilon_{k} f\left(z, x^{k+1}\right)-\lambda_{k} h\left(z, x^{k+1}\right)-\gamma D\left(x^{k+1}, x^{k}\right) .
\end{aligned}
$$

Because $w-p \in \partial h_{z}(z)$, it follows that $h\left(z, x^{k+1}\right) \geq\left\langle w-p, x^{k+1}-z\right\rangle$. Hence,

$$
\begin{aligned}
D\left(z, x^{k+1}\right)+\frac{1}{2} \lambda_{k} \varepsilon_{k} f\left(z, x^{k+1}\right) \leq & D\left(z, x^{k}\right)-\frac{1}{2} \lambda_{k} \varepsilon_{k} f\left(z, x^{k+1}\right) \\
& +\lambda_{k}\left\langle w-p, z-x^{k+1}\right\rangle-\gamma D\left(x^{k+1}, x^{k}\right) .
\end{aligned}
$$

Rewriting the last inequality, we obtain

$$
\begin{aligned}
& D\left(z, x^{k+1}\right)+\frac{\lambda_{k} \varepsilon_{k}}{2} f\left(z, x^{k+1}\right) \\
& \quad \leq D\left(z, x^{k}\right)-\gamma D\left(x^{k+1}, x^{k}\right)+\lambda_{k}\left\langle w, z-x^{k+1}\right\rangle \\
& \quad+\frac{\lambda_{k} \varepsilon_{k}}{2}\left[\left\langle\frac{2 p}{\varepsilon_{k}}, x^{k+1}\right\rangle-f\left(z, x^{k+1}\right)-\left\langle\frac{2 p}{\varepsilon_{k}}, z\right\rangle-\delta_{S(f, K)}(z)\right] .
\end{aligned}
$$

Since $z \in S(f, K), \delta_{S(f, K)}(z)=0$. Moreover, we have

(i) $\frac{2 p}{\varepsilon_{k}} \in \partial \delta_{S(f, K)}(z)=\mathcal{N}_{S(f, K)}(z)$, so $\delta_{S(f, K)}^{*}\left(\frac{2 p}{\varepsilon_{k}}\right)=\sigma_{S(f, K)}\left(\frac{2 p}{\varepsilon_{k}}\right)$;

(ii) $\left\langle\frac{2 p}{\varepsilon_{k}}, x^{k+1}\right\rangle-f_{z}\left(x^{k+1}\right) \leq f_{z}^{*}\left(\frac{2 p}{\varepsilon_{k}}\right)$. 
Therefore, the desired result follows by combining (i) and (ii) with the last inequality.

Theorem 6.3. Assume that $S(h, S(f, K)) \neq \emptyset$. For all $x^{0} \in K$, we have the following:

(i) The sequence $\left\{x^{k}\right\}$ generated by Algorithm 1 is well defined.

(ii) For all $z \in S(h, S(f, K))$,

$$
D\left(z, x^{k+1}\right) \leq D\left(z, x^{k}\right)+\frac{1}{2} \lambda_{k} \varepsilon_{k}\left[f_{z}^{*}\left(\frac{2 p}{\varepsilon_{k}}\right)-\sigma_{S(f, K)}\left(\frac{2 p}{\varepsilon_{k}}\right)\right] .
$$

(iii) If ( $\mathcal{H})$ holds, then for all $z \in S(h, S(f, K))$

(a) $\lim _{k \rightarrow \infty} D\left(z, x^{k}\right)$ exists;

(b) the sequence $\left\{x^{k}\right\}$ is bounded;

(c) $\lim _{k \rightarrow \infty} D\left(x^{k+1}, x^{k}\right)=0$ and $\lim _{k \rightarrow \infty}\left(x^{k+1}-x^{k}\right)=0$;

(d) $\sum_{k=1}^{\infty} \varepsilon_{k} f\left(z, x^{k+1}\right)<\infty$.

Proof. Item (i) follows from Theorem 5.5. For item (ii), take an arbitrary $z \in$ $S(h, S(f, K))$, i.e., $h(z, y) \geq 0$ for all $y \in S(f, K)$. Hence,

$$
z \in \operatorname{argmin}_{y \in S(f, K)} h_{z}(y) \Longleftrightarrow 0 \in \partial\left(h_{z}+\delta_{S(f, K)}\right)(z) .
$$

Taking into account that $z \in S(f, K),-\gamma D\left(x^{k+1}, x^{k}\right) \leq 0$ and letting $w=0$ in (6.3), item (ii) follows. As assumption $(\mathcal{H})$ holds, by Lemma $6.1 \lim _{k \rightarrow \infty} D\left(z, x^{k}\right)$ exists. In particular, $\left\{D\left(z, x^{k}\right): k \in \mathbb{N}\right\}$ is a bounded set and, from property (H3), we conclude that the sequence $\left\{x^{k}\right\}$ is also bounded. This proves items (a) and (b). Given that $z \in S(f, K)$ and letting $w=0$ in (6.3), we have

$$
\gamma D\left(x^{k+1}, x^{k}\right) \leq-D\left(z, x^{k+1}\right)+D\left(z, x^{k}\right)+\frac{1}{2} \lambda_{k} \varepsilon_{k}\left[f_{z}^{*}\left(\frac{2 p}{\varepsilon_{k}}\right)-\sigma_{S(f, K)}\left(\frac{2 p}{\varepsilon_{k}}\right)\right] .
$$

Thus, $\lim _{k \rightarrow \infty} D\left(x^{k+1}, x^{k}\right)=0$ follows from assumption $(\mathcal{H})$ together with item (a), and the completion of the proof of item (c) is obtained from Proposition 4.4. Using again $w=0$ in (6.3), since $\liminf \operatorname{in}_{k \rightarrow \infty} \lambda_{k}>0$, there exists $\theta>0$ such that

$$
\theta \varepsilon_{k} f\left(z, x^{k+1}\right) \leq D\left(z, x^{k}\right)-D\left(z, x^{k+1}\right)+\frac{1}{2} \lambda_{k} \varepsilon_{k}\left[f_{z}^{*}\left(\frac{2 p}{\varepsilon_{k}}\right)-\sigma_{S(f, K)}\left(\frac{2 p}{\varepsilon_{k}}\right)\right] .
$$

Finally, item (d) follows by combining item (a) and assumption $(\mathcal{H})$.

Lemma 6.4. Assume $(\mathcal{H})$ holds, $S(h, S(f, K)) \neq \emptyset$, and let $\left\{x^{k}\right\}$ be the sequence generated by Algorithm 1. If $\lim _{j \rightarrow \infty} x^{k_{j}}=x^{*}$, then

(a) $\lim _{j \rightarrow \infty} x^{k_{j}+1}=x^{*}$,

(b) $x^{*} \in S(f, K)$,

(c) $x^{*} \in S(h, S(f, K))$.

Proof. From the triangular inequality, we have

$$
\left\|x^{k_{j}+1}-x^{*}\right\| \leq\left\|x^{k_{j}+1}-x^{k_{j}}\right\|+\left\|x^{k_{j}}-x^{*}\right\|, \quad j=0,1, \ldots,
$$

and item (a) follows. Now, the definition of $x^{k_{j}+1}$ combined with property (H4), yields

$f\left(x^{k_{j}+1}, y\right)+\frac{1}{\varepsilon_{k_{j}}} h\left(x^{k_{j}+1}, y\right)+\frac{1}{\lambda_{k_{j}} \varepsilon_{k_{j}}}\left[D\left(y, x^{k_{j}}\right)-D\left(y, x^{k_{j}+1}\right)-\gamma D\left(x^{k_{j}+1}, x^{k_{j}}\right)\right] \geq 0$ 
for all $y \in K$ and some $\gamma>0$. On the other hand, as $(\mathcal{H})$ and $(H 1)$ happen, from item (ii) of Theorem 6.3 it is easy to see that

$$
\limsup _{j \rightarrow \infty}\left[D\left(y, x^{k_{j}}\right)-D\left(y, x^{k_{j}+1}\right)-\gamma D\left(x^{k_{j}+1}, x^{k_{j}}\right)\right]=0 .
$$

Because $f$ and $h$ satisfy ( $L 2)$, taking the limsup as $j$ goes to $\infty$ in (6.5) and using the fact that $\liminf _{k \rightarrow \infty} \lambda_{k}>0, \lim _{k \rightarrow \infty} \varepsilon_{k}=\infty$, and (6.6) happens, we obtain

$$
f\left(x^{*}, y\right) \geq 0, \quad y \in K,
$$

which proves item (b). The proof of item (c) follows by using arguments similar to those in item (b) and the fact that $f(x, y)=0$ for all $x, y \in S(f, K)$.

\subsection{Convergence to a bilevel equilibrium.}

Definition 6.5. A sequence $\left\{z^{k}\right\} \subset \mathbb{R}^{n}$ is said to be quasi-Fejér convergent to a set $U \neq \emptyset$ with respect to the generalized proximal distance $(d, D)$, if there exists a nonnegative summable sequence $\left\{\gamma_{k}\right\}$ such that, for each $k \in \mathbb{N}$,

$$
D\left(z^{k+1}, u\right) \leq D\left(z^{k}, u\right)+\gamma_{k}, \quad u \in U .
$$

The next result is important to establish the convergence of the sequence generated by Algorithm 1.

Lemma 6.6. If $\left\{z^{k}\right\} \subset \mathbb{R}^{n}$ is quasi-Fejér convergent to a set $U \neq \emptyset$ with respect to the generalized proximal distance $(d, D)$, then $\left\{z^{k}\right\}$ is bounded. If a cluster point $z$ of $\left\{z^{k}\right\}$ belongs to $U$, then $\lim _{k \rightarrow \infty} z^{k}=z$.

Proof. The proof is presented by Iusem, Svaiter, and Teboulle in [38].

Let us show that the whole sequence $\left\{x^{k}\right\}$ converges to a solution of (1.1).

ThEOREM 6.7. Under the assumptions of Theorem 6.3, the whole sequence $\left\{x^{k}\right\}$, generated by Algorithm 1, converges to a solution of (1.1).

Proof. Inequality (6.4) combined with assumption $(\mathcal{H})$ allows us to conclude that $\left\{x^{k}\right\}$ is quasi-Fejér convergent to the set $S(h, S(f, K))$ with respect to the generalized proximal distance $(d, D)$. Hence, $\left\{x^{k}\right\}$ is bounded (it follows from Lemma 6.6). Let $x^{*}$ be a cluster point of $\left\{x^{k}\right\}$. Item (c) in Lemma 6.4 implies that $x^{*} \in S(h, S(f, K))$ and the conclusion of the proof follows by using again Lemma 6.6.

6.3. Examples. In this section, we present two classes of problems for which the assumptions of our main results are verified. For the first class of examples, we need the following definition, which has appeared, for example, in [39]. Let $\psi: K \times K \rightarrow \mathbb{R}$ be a bifunction and consider the equilibrium problem associated with $\psi$ : find $x^{*} \in K$ such that $\psi\left(x^{*}, y\right) \geq 0$ for every $y \in K$. A solution $\hat{x} \in S(\psi, K)$ is said to be a trivial solution for the equilibrium problem if $\psi(\hat{x}, y)=0$ for every $y \in K$.

6.3.1. Fixed point in the lower level problem. Consider the bilevel problem (1.1) where each $\hat{x} \in S(f, K)$ is a trivial solution for the lower level problem. This holds, for example, when $f(x, y):=\langle x-T x, y-x\rangle$, where $T: K \rightarrow K$ is an operator. Note that, in this case, $f$ satisfies $(L 1)-(L 3)$ and, if $T$ is a nonexpansive operator, 
then $f$ also satisfies $(L 4)$. As noted in [9], Assumption $\mathcal{H}$ is verified and the bilevel problem (1.1) reduces to:

$$
\text { find } \quad \bar{x} \in \operatorname{Fix}(T) \quad \text { such that } h(\bar{x}, y) \geq 0, \quad y \in \operatorname{Fix}(T),
$$

where $\operatorname{Fix}(T):=\{x \in K: T(x)=x\}$. Indeed, it is possible to show that Assumption $\mathcal{H}$ holds whenever each $\hat{x} \in S(f, K)$ is a trivial solution for the lower level problem.

6.3.2. Minimization in the lower level problem. Let $\Psi: K \rightarrow \mathbb{R}$ be a convex and lsc function and assume that $f(x, y):=\Psi(y)-\Psi(x)$. In this case, $S(f, K)=\operatorname{argmin}_{x \in K} \Psi(x)$ and the bilevel problem (1.1) reduces to

find $\quad \bar{x} \in \operatorname{argmin}_{x \in K} \Psi(x) \quad$ such that $h(\bar{x}, y) \geq 0, \quad y \in \operatorname{argmin}_{x \in K} \Psi(x)$.

For an appropriate choice of $h$, this problem can be seen as a hierarchical minimization problem; see section 3 . It is easy to see that $f$ satisfies $(L 1)-(L 4)$. Now, let us suppose that $\operatorname{argmin}_{x \in k} \Psi(x) \neq \emptyset, \Psi(x)=0$ for all $x \in \operatorname{argmin}_{x \in k} \Psi(x)$, and assume that for some $\theta>0$ and $m>1$, we have

$$
\Psi_{0}(y) \geq \frac{\theta}{m} \operatorname{dist}(y, S(f, K))^{m}, \quad y \in \mathbb{R}^{n},
$$

where $\Psi_{0}(y)=\Psi(y)$ if $y \in K$ and $\Psi_{0}(y)=\infty$ if $y \notin K$. Thus, using the definition of $\operatorname{dist}(\cdot, S(f, K))$ and simple algebraic manipulations, from (6.7) we obtain, for $y \in \mathbb{R}^{n}$

$$
\Psi_{0}(y) \geq \inf _{x \in \mathbb{R}^{n}}\left(\frac{\theta}{m}\|x-y\|^{m}+\delta_{S(f, K)}(x)\right)=\left(\frac{\theta}{m}\|\cdot\|^{m} \square \delta_{S(f, K)}\right)(y),
$$

where $\square$ denotes the inf-convolution. Let us denote by $\Psi_{0}^{*}$ the conjugate function of $\Psi_{0}$. It is known that $\left(\frac{\theta}{m}\|\cdot\|^{m} \square \delta_{S(f, K)}\right)^{*}(y)=\left(\frac{\theta}{m}\|\cdot\|^{m}\right)^{*}(y)+\left(\delta_{S(f, K)}\right)^{*}(y)$ and $\left(\frac{\theta}{m}\|\cdot\|^{m}\right)^{*}(y)=\frac{\theta^{1-s}}{s}\|y\|^{s}$ for $s>0$ such that $\frac{1}{m}+\frac{1}{s}=1$; see, for instance, [37, pp. 106 and 145] (in the first equality we use that $S(f, K)$ is a convex set). Hence, for $s>0$ such that $\frac{1}{m}+\frac{1}{s}=1,(6.8)$ implies

$$
\Psi^{*}(y) \leq\left(\frac{\theta}{m}\|\cdot\|^{m} \square \delta_{S(f, K)}\right)^{*}(y)=\frac{\theta^{1-s}}{s}\|y\|^{s}+\sigma_{S(f, K)}(y), \quad y \in \mathbb{R}^{n},
$$

where the first inequality follows by using an elementary property of the conjugate of convex functions; see [37, p. 104]. In particular, we note that if the sequences $\left\{\lambda_{k}\right\}$ and $\left\{\varepsilon_{k}\right\}$ are chosen such that $\sum_{k=1}^{\infty} \frac{\lambda_{k}}{\varepsilon_{k}^{s-1}}<\infty$, then Assumption $(\mathcal{H})$ holds. This becomes evident when taking $p \in \mathcal{R}\left(\mathcal{N}_{S(f, K)}\right)$ and $y=\left(\frac{2 p}{\varepsilon_{k}}\right)$ in (6.9). Let us point out that the case $m=2$ was considered in [22]. Besides, we note that condition (6.7) also appeared within [19] in the context of hierarchical minimization.

Remark 6.2. In both examples $S(f, K)$ is assumed to be nonempty. Then, condition $(L 5)$ is verified because $f$ is, in each case, a monotone bifunction.

7. Application to stability and change dynamics of a hierarchical organization. Using a specific, but important VR variational structure, the algorithm given in the previous section shows when a worthwhile stay and change dynamic (transition) $x^{k+1} \in W_{e_{k}, \varepsilon_{k+1}, \xi_{k+1}}\left(x^{k}\right), k \in \mathbb{N}$, of a hierarchical organization, approaches 
(converges) and ends (reaches) in a variational trap, worthwhile to reach (an aspiration point), and not worthwhile to leave (a stationary trap). More precisely, given the previous period $k$, the current period $k+1$, and the future period $k+2$, we use the nice variational BEP formulation, to modelize in the context of the VR approach, the dynamic of a leader-follower relationship as a succession of worthwhile temporary stays and changes. At the behavioral level, this problem is really challenging, because its core hypothesis takes for granted that the environment, both internal and external to the organization, is changing, which is presently the general case in our rapidly changing world. Each period, as past experience $e_{k}$ evolves, the current sharing rule $\varepsilon_{k+1}$, between advantages to change of, both, the leader and the follower, and the variable worthwhile to change ratio $\xi_{k+1}=1 / \lambda_{k}$, must be adapted to each period. Then, at the behavioral level, the great merit of our work is to be able to show how, starting from an uncomfortable initial position, the leader can use an adaptive process with the hope of reaching a desired end, following an acceptable/worthwhile transition, both for him and for the follower. This worthwhile to change process balances, for each period, in an adaptive way, on the one side, the advantages of changing for the leader and the follower, and, on the other side, the weighted advantages to change of both of them to their inconveniences to change, to end in a variational trap.

This process (our proximal point algorithm; see section 6.1) follows a worthwhile to change transition consisting of a succession of weak stationary traps, with each of them not being worthwhile to leave, given the current environment. Let us show that each of these successive weak stationary traps is also worthwhile reaching from the previous trap. This shows that our algorithm successfully modelizes a succession of variational traps, worthwhile to reach from the previous trap, but not worthwhile to leave. More precisely, given $\bar{x} \in X$, Lemma 5.3 shows that if $f$ and $h$ satisfy conditions $(L 1)-(L 4)$, then $f_{k}$ defined in (6.1) also satisfies conditions $(L 1)-(L 4)$. In particular, if $f_{k}(x, y) \geq 0$, we have $f_{k}(y, x) \leq 0$. Then, suppose that, in the current period $k+1$, $f_{k}\left(x^{k+1}, y\right) \geq 0$ for all $y \in K$. This means that, in the current period, $x=x^{k+1}$ is a weak stationary trap, i.e., it is not worthwhile to move from $x=x^{k+1}$ to any other position $y \in K$. This implies, in particular, that it is not worthwhile returning to the previous position $y=x^{k}$, i.e., $f_{k}\left(x^{k+1}, x^{k}\right) \geq 0$. Then, $(L 4)$ implies that $f_{k}\left(x^{k}, x^{k+1}\right) \leq 0$, i.e., it is worthwhile to directly move from $x^{k}$ to $x^{k+1}$. This shows that $x^{k+1}$ is a weak variational trap, worthwhile to reach from the last past position $x^{k}$, but not worthwhile to leave. Notice that, in the application, $(L 4)$ is satisfied by the two functions $f$ and $h$, because they are separable, as we have supposed in the example. However, the current variational trap of the current period ceases to be a variational trap in the next period, because the environment is changing. The proximal point algorithm given in (6.1) defines an acceptable (worthwhile) stop and goes dynamic where, during each period, the leader and the follower can bargain. Setting, for each period, bounded sharing rules (which can change within bounds), and moving from a current weak variational trap to the next, allows convergence of the leaderfollower relationship to a stable hierarchical position, a BEP equilibrium (a weak stationary trap, with no inertia). In this way, the present algorithm, using the VR approach, modelizes a habituation/routinization relationship, such that, step by step, gradually, the organization implements an increasingly similar collective action. When a worthwhile to change process converges to a weak variational trap, this variational formulation offers a model of routine formation, ending in a collective trap, as the end point of a path of worthwhile temporary stays and changes.

To summarize, the striking point is that, in our VR model, the formation of habitual collective actions can occur even in a nonstationary environment. In each 
period, bargaining over payoffs destabilizes the current weak variational trap, and the process proceeds to the next weak variational trap. However, in each period, the collective action becomes increasingly similar, ending in a routinized collective action, a hierarchical (bilevel) equilibrium, which is stable, even with no inconveniences to change at the end! This shows that such an equilibrium defines, at the end, a kind of very stable solution.

Remark 7.1. The behavioral content of the standard assumptions given in section 5.1 can be described as follows:

(i) (L1) indicates that the advantages (or losses) to change from $x$ to $y$ are zero for any stay;

(ii) (L2) and (L3) refer to regularity assumptions, which are natural assumptions for the advantages to change function in the VR approach;

(iii) (L4) means that if there is an advantage to change from $x$ to $y$, there is a disadvantage to change from $y$ to $x$ (a no regret condition);

(iv) (L5) means that for any unbounded sequence of actions $\left\{y^{k}\right\}$, there exists an aspiration point $u$ which the agent aims to reach, starting from any position $y^{k}, k \geq k_{0}$.

The fact that ( $L 5)$ supposes the existence of aspiration points, confirms the close relationship between proximal algorithms and, more generally, the variational principles of the VR approach, inspired by behavioral science principles, where an aspiration point defines one side of a variational trap (worthwhile to reach from some, or any point, of the sequence), the other side being the stability aspect (an equilibrium, not worthwhile to leave). This offers a striking new point of view.

\section{REFERENCES}

[1] J. Schumpeter, The economy as a whole: The seventh chapter to Schumpeter's theory of economic development (1912), Ind. Innov., 9 (2002), pp. 93-145.

[2] J. Schumpeter, New translations from theorie der wirtschaftlichen Entwicklung, Am. J. Econ. Sociol., 61 (2002), pp. 405-437.

[3] R. Nelson And S. Winter, An Evolutionary Theory of Economic Change, Belknap Press/Harvard University Press, Cambridge, MA, 1982.

[4] C. LeANNA AND B. BARRY, Stability and change as simultaneous experiences in organizational life, Acad. Manag. Rev., 25 (2000), pp. 753-759.

[5] A. Soubeyran, Variational Rationality, a Theory of Individual Stability and Change: Worthwhile and Ambidextry Behaviors, preprint, GREQAM, Aix Marseille University, Marseille 2009.

[6] A. Soubeyran, Variational Rationality and the Unsatisfied Man: Routines and the Course Pursuit Between Aspirations, Capabilities, Beliefs, preprint, GREQAM, Aix Marseille University, Marseille, 2010.

[7] O. Chadli, Z. Chbani, and H. Riahi, Equilibrium problems with generalized monotone bifunctions and applications to variational inequalities, J. Optim. Theory Appl., 105 (2000), pp. 299-323.

[8] A. Moudafi, Proximal methods for a class of bilevel monotone equilibrium problems, J. Global Optim., 47 (2010), pp. 287-292.

[9] Z. ChBAni AND H. RiAhI, Weak and strong convergence of prox-penalization and splitting algorithms for bilevel equilibrium problems, Numer. Algebra Control Optim., 2 (2013), pp. 353-366.

[10] D. MuU And W. Oettli, Convergence of an adaptive penalty scheme for finding constrained equilibria, Nonlinear Anal. Theory, 18 (1992), pp. 1159-1161.

[11] H. Von Stackelberg, The Theory of the Market Economy, Oxford University Press, New York, 1952.

[12] S. Dempe And J. DutTA, Is bilevel programming a special case of a mathematical program with complementarity constraints?, Math. Program., Ser. A 131 (2012), pp. 37-48. 
[13] X. P. Ding, Auxiliary principle and algorithm for mixed equilibrium problems and bilevel mixed equilibrium problems in Banach spaces, J. Optim. Theory Appl., 146 (2010), pp. 347-357.

[14] L. Q. ANH, P. Q. Khanh, AND D. T. M. VAN, Well-posedness under relaxed semicontinuity for bilevel equilibrium and optimization problems with equilibrium constraints, J. Optim. Theory Appl., 153 (2012), pp. 42-59.

[15] J. Chen, Z. WAN, AND Y. J. CHO, The existence of solutions and well-posedness for bilevel mixed equilibrium problems in Banach spaces, Taiwanese J. Math., 17 (2013), pp. 725-748.

[16] J. Q. LuO, J. S. PANG, AND D. RALPh, Mathematical Programs with Equilibrium Constraints, Cambridge University Press, Cambridge, UK, 1996.

[17] M. A. Migdalas, P. Pardalos, And P. Varbrand, (eds) Multilevel Optimization: Algorithms and Applications, Kluwer, Dordrecht, 1997.

[18] J. BRACKEn AND J. T. MCGILl, Mathematical programs with optimization problems in the constraints, Oper. Res., 21 (1973), pp. 37-44.

[19] A. САвот, Proximal point algorithm controlled by a slowly vanishing term: Applications to hierarchiccal minimization, SIAM J. Optim., 15 (2005), pp. 555-572.

[20] F. FAcchinei, J. S. PANG, G. Scutari, and L. Lampariello, VI-constrained hemivariational inequalities: Distributed algorithms and power control in ad-hoc networks, Math. Program. Ser. A, 145 (2014), pp. 59-96.

[21] A. Moudafi, Proximal point algorithm extended for equilibrium problems, J. Natur. Geom., 15 (1999), pp. 91-100.

[22] H. Attouch, M.-O. Czarnecki, and J. Peypouquet, Prox-penalization and splitting methods for constrained variational problems, SIAM J. Optim., 21 (2011), pp. 149-173.

[23] H. Аtтouch, M.-O. Czarnecki, and J. Peypouquet, Coupling forward-backward with penalty schemes and parallel splitting for contrained variational inequalities, SIAM J. Optim., 21 (2011), pp. 1251-1274.

[24] B. Van Dinh And L. Dung Muu, On penalty and gap function methods for bilevel equilibrium problems, J. Appl. Math., 2011 (2011), 646452, doi:10.1155/2011/646452.

[25] P. J. S. Silva And J. ECKSTein, Double-regularization proximal methods, with complementarity applications, Comput. Optim. Appl., 33 (2006), pp. 115-156.

[26] A. Auslender And M. Teboulle, Interior gradient and proximal methods for convex and conic optimization, SIAM J. Optim., 16 (2006), pp. 697-725.

[27] R. BURACHIK AND J. DUtTA,Inexact proximal point methods for variational inequality Problems, SIAM J. Optim., 20 (2010), pp. 2653-2678.

[28] A. N. Iusem, G. Kassay, And W. Sosa, Certain conditions for the existence of solutions of equilibrium problems, Math. Program., 116 (2009), pp. 259-273.

[29] E. Blum And W. OetTli, From optimization and variational inequality to equilibrium problems, Math. Stud., 63 (1994), pp. 127-149.

[30] K. FAN, A minimax inequality and applications, Inequalities III, O. Shisha, ed., Academic, New York, 1972, pp. 103-113.

[31] R. BURACHIK AND G. Kassay, On a generalized proximal point method for solving equilibrium problems in Banach spaces, Nonlinear Anal. Theory, 75 (2012), pp. 6456-6464.

[32] P. P. B. EgGermont, Multiplicative iterative algorithms for convex programming, Linear Algebra Appl., 130 (1990), pp. 25-42.

[33] P. Tseng And D. P. Bertsekas, On the convergence of the exponential multiplier methods for convex programming. Math. Program., 60 (1993), pp. 1-19.

[34] A. N. Iusem And M. Teboulle, Convergence rate analysis of nonquadratic proximal methods for convex and linear programming, Math. Oper. Res., 20 (1995), pp. 657-677.

[35] J. X. Cruz Neto, A. N. Iusem, O. P. Ferreira, and R. D. C. Monteiro, Dual convergence of the proximal point method with Bregman distances for linear programming, Optim. Methods Softw., 22 (2007), pp. 339-360.

[36] B. T. POLYAK, Introduction to Optimization, Transl. Ser. Math. Eng., Optimization Software, New York, 1987.

[37] R. T. Rockafellar, Convex Analysis. Princeton University Press, Princeton, NJ, 1970

[38] A. N. Iusem, B. Svaiter, And M. Teboulle, Entropy-like proximal methods in convex programming, Math. Oper. Res., 19 (1994), pp. 790-814.

[39] M. Bianchi And R. PIni, Coercivity conditions for equilibrium problems, J. Optim. Theory Appl., 124 (2005), pp. 79-92. 